\title{
Entanglement of several blocks in fermionic chains
}

\author{
Filiberto Ares* \\ Departamento de Física Teórica, Universidad de Zaragoza, 50009 Zaragoza, Spain \\ José G. Esteve ${ }^{\dagger}$ and Fernando Falceto ${ }^{\ddagger}$ \\ Departamento de Física Teórica, Universidad de Zaragoza, 50009 Zaragoza, Spain \\ and Instituto de Biocomputación y Física de Sistemas Complejos (BIFI), 50009 Zaragoza, Spain
}

(Received 16 July 2014; published 15 December 2014)

\begin{abstract}
In this paper we propose an expression for the entanglement entropy of several intervals in a stationary state of a free, translational invariant Hamiltonian in a fermionic chain. We check numerically the accuracy of our proposal and conjecture a formula for the asymptotic behavior of principal submatrices of a Toeplitz matrix.
\end{abstract}

DOI: 10.1103/PhysRevA.90.062321

PACS number(s): 03.67.Mn, 05.30.Fk, 05.70.Jk

\section{INTRODUCTION}

For the last years a considerable effort has been invested to understand the entanglement of quantum systems. This is, in Schrödinger's words, the characteristic trait of quantum mechanics [1]. Moreover, the study of entanglement has a considerable interest from many perspectives, ranging from condensed-matter physics [2,3] or quantum field theory $[4,5]$ to black-hole physics [6] and the holographic principle [7].

One of the preferred magnitudes to characterize entanglement is the Rényi entropy of the reduced state, that provides information on the full entanglement spectrum. Let us consider a bipartite system such that its Hilbert space can be written as the tensor product $\mathcal{H}=\mathcal{H}_{X} \otimes \mathcal{H}_{Y}$, of the Hilbert space of subsystems $X$ and $Y$ in which we have divided it. If $\rho$ is the density matrix that describes the state of the whole system, the Rényi entropy of $X$ is defined as

$$
S_{\alpha}(X)=\frac{1}{1-\alpha} \ln \operatorname{Tr}\left(\rho_{X}^{\alpha}\right)
$$

where $\rho_{X}=\operatorname{Tr}_{Y}(\rho)$ is the reduced density matrix of $X$ with $\operatorname{Tr}_{Y}$ denoting the partial trace to the complementary subsystem $Y$. In the limit $\alpha \rightarrow 1$, we obtain von Neumann entropy,

$$
S_{1}(X)=-\operatorname{Tr}\left(\rho_{X} \ln \rho_{X}\right) .
$$

If the system is in a pure state $|\Psi\rangle$, then $\rho=|\Psi\rangle\langle\Psi|$ and $S_{\alpha}(X)=S_{\alpha}(Y)$. In that case, this quantity provides a very appropriate measure of the degree of entanglement between $X$ and $Y$ in the state $|\Psi\rangle$ [8]. Furthermore, it encodes universal properties of extended systems in the neighborhood of quantum critical points [2].

The study of $S_{\alpha}(X)$ for fermionic chains is specially interesting and simple because on the one side, they can be mapped to spin chains by means of a nonlocal Jordan-Wigner transform and, on the other side, we can apply both analytical techniques and efficient numerical algorithms. In this respect, much work has been done when $X$ is a single interval. In this case, a general result [9] for the eigenstates of a free,

\footnotetext{
*ares@unizar.es

†Corresponding author: esteve@unizar.es

${ }^{\ddagger}$ falceto@unizar.es
}

translational invariant Hamiltonian can be obtained using the fact that the correlation matrix of an interval is of the Toeplitz type (this property was first noticed by Jin and Korepin [10] for the ground state, and it is also applied in, e.g., [11-14]). There are other different approaches. In particular, conformal field theory (CFT) is a powerful tool for the ground-state entanglement entropy when the chain is described by a local and critical Hamiltonian [15-17] and it can be extended to excited states too $[18,19]$. On the numerical side, we can reduce the complexity of computing the Rényi entropy which, in principle, grows exponentially with the size of the subsystem to a polynomial dependence. This is possible thanks to the relationship obtained in $[17,20]$ between the density matrix and the two-point correlation functions for situations, like ours, in which Wick factorization holds.

A natural extension of the previous works is to consider a subsystem $X$ composed of disjoint intervals. There are some recent papers where this problem is addressed for the ground state of a local and critical Hamiltonian, which can be analyzed using CFT [21-26]. If we try to apply the previous technique to this case we find that, although Wick factorization still holds and therefore the complexity of the computation grows polynomially, the corresponding matrix for several blocks is no longer of the Toeplitz type and the asymptotic expansion for its determinant is not known in the literature, so far.

In this work, inspired by the previous analytical results and some particular examples, we conjecture a general asymptotic expression of $S_{\alpha}(X)$ for the eigenstates of a free, translational invariant fermionic Hamiltonian when $X$ is composed by several disjoint blocks. We check our hypothesis numerically and trace back its origin to a conjecture on the determinant of a principal submatrix of a Toeplitz matrix.

The paper is organized in the following fashion. In the next section, we introduce the notation and review the results for the entropy of a single interval. In Sec. III, we recall the results predicted by CFT for two disjoint intervals in the ground state of local theories and we propose a conjecture for a general eigenstate. We check it numerically in Sec. IV, while in Sec. V we generalize our formula for an arbitrary number of disjoint intervals and conjecture an asymptotic expression for the determinant of a submatrix of a Toeplitz matrix. Finally in Sec. VI we collect our conclusions and possible continuations of our work. 


\section{ENTANGLEMENT ENTROPY AND TOEPLITZ DETERMINANTS}

We consider a chain of $N$ identical spinless fermions with $a_{n}$ and $a_{n}^{\dagger}, n=1, \ldots, N$, representing respectively the annihilation and creation operator for the site $n$. The only nonvanishing anticommutation relations are

$$
\left\{a_{n}, a_{m}^{\dagger}\right\}=\delta_{n m} .
$$

Furthermore, we shall assume periodic boundary conditions: $a_{N+1} \equiv a_{1}$.

We consider the eigenstates of a free, translational invariant Hamiltonian,

$$
\left|\Psi_{\mathcal{K}}\right\rangle=\prod_{k \in \mathcal{K}} b_{k}^{\dagger}|0\rangle
$$

where $b$ operators are the discrete Fourier transform of $a$ operators,

$$
b_{k}=\frac{1}{\sqrt{N}} \sum_{n=1}^{N} e^{2 \pi i k n / N} a_{n}, \quad k=-N / 2, \ldots, N / 2-1,
$$

which also satisfy the canonical anticommutation relations. The ket $|0\rangle$ represents the vacuum state in the Fock space, $\mathcal{K} \subset$ $\{-N / 2, \ldots, N / 2-1\}$ is a particular set of occupied modes, and

$$
b_{k}^{\dagger}=\frac{1}{\sqrt{N}} \sum_{n=1}^{N} e^{-2 \pi i k n / N} a_{n}^{\dagger}
$$

is the adjoint of $b_{k}$.

We divide the chain into two subsets $X$ and $Y=$ $\{1, \ldots, N\} \backslash X$. Adapted to this decomposition we can factor out the Hilbert space $\mathcal{H}=\mathcal{H}_{X} \otimes \mathcal{H}_{Y}$. The goal is to study the entanglement between these subsystems.

In order to do that we must construct the reduced density matrix of each subsystem, that in general does not correspond to a pure state, and compute its Rényi entropy. As it was discussed before, for pure states, the entropy of a subsystem coincides with that of the complementary one and provides a measurement for the entanglement between them. Once we have obtained the reduced density matrix, we can compute its Rényi entropy. However, considering that the dimension of $\mathcal{H}_{X}$ is $2^{|X|}$, the computational time grows exponentially with the size $|X|$ of the subsystem.

Fortunately, there exists an algorithm [17,20] that can be applied in some cases and allows us to reduce the exponential growth to a potential one. According to it, if the reduced density matrix satisfies the Wick decomposition property, i.e., the $n$-point functions factor out into two-point functions (see $[9,20])$, the full reduced density matrix $\rho_{X}$, of dimension $2^{|X|}$ can be obtained from the two-point correlation matrix, whose dimension is $|X|$. This dramatic gain of computational power allows us to deal with larger subsystems $X$ without exhausting the computational capabilities. This is essential for us, as we will be interested in the asymptotic behavior of the entanglement entropy for large values of the size of the subsystem.

It is easy to show that the Wick decomposition property, for the reduced density matrix, follows from the same one for the full density matrix. And the latter enjoys this property for any

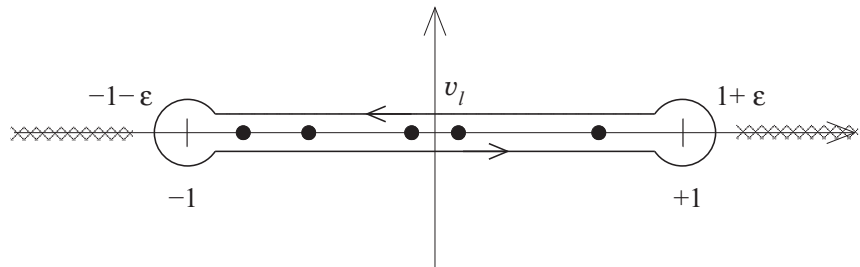

FIG. 1. Contour of integration, cuts, and poles for the computation of $S_{\alpha}(X)$. The cuts for the function $f_{\alpha}$ extend to $\pm \infty$.

pure state corresponding to a Slater determinant, like the one in (1).

For these states the full density matrix, $\rho=\left|\Psi_{\mathcal{K}}\right\rangle\left\langle\Psi_{\mathcal{K}}\right|$, preserves the total fermionic number and therefore $\operatorname{Tr}\left(\rho a_{n} a_{m}\right)=$ $\operatorname{Tr}\left(\rho a_{n}^{\dagger} a_{m}^{\dagger}\right)=0$. Evidently, this property is also fulfilled by the reduced density matrices.

In that case it will be useful to introduce the commutator expectation value matrix

$$
\left(V_{X}\right)_{n m}=\operatorname{Tr}\left(\rho\left[a_{n}^{\dagger}, a_{m}\right]\right), \quad n, m \in X
$$

in terms of which the Rényi entropy reads [9]

$$
\begin{aligned}
S_{\alpha}(X) & =\frac{1}{1-\alpha} \operatorname{Tr} \ln \left[\left(\frac{I-V_{X}}{2}\right)^{\alpha}+\left(\frac{I+V_{X}}{2}\right)^{\alpha}\right] \\
& =\lim _{\varepsilon \rightarrow 0^{+}} \frac{1}{2 \pi i} \oint_{\mathcal{C}} f_{\alpha}(1+\varepsilon, \lambda) \frac{d \ln \operatorname{det}\left(\lambda I-V_{X}\right)}{d \lambda} d \lambda .
\end{aligned}
$$

In the second expression, we have made use of the Cauchy's residue theorem, with

$$
f_{\alpha}(x, y)=\frac{1}{1-\alpha} \ln \left[\left(\frac{x+y}{2}\right)^{\alpha}+\left(\frac{x-y}{2}\right)^{\alpha}\right],
$$

and $\mathcal{C}$ the contour depicted in Fig. 1 that surrounds all the poles of the logarithmic derivative of the determinant, i.e., the eigenvalues, $v_{l}$, of $V_{X}$.

For the state $\left|\Psi_{\mathcal{K}}\right\rangle$, the matrix $V_{X}$ can be written

$$
\left(V_{X}\right)_{n m}=\frac{1}{N}\left(\sum_{k \in \mathcal{K}} e^{i \theta_{k}(n-m)}-\sum_{k \notin \mathcal{K}} e^{i \theta_{k}(n-m)}\right),
$$

with $\theta_{k}=2 \pi k / N$.

We are interested in the large $N$ (thermodynamic) limit, in which case the role played by $\mathcal{K}$ is taken by a density $g(\theta)$, such that

$$
\begin{aligned}
& \frac{1}{2 \pi} \int_{-\pi}^{\pi} g(\theta) e^{i(n-m) \theta} d \theta \\
& \quad=\lim _{N \rightarrow \infty} \frac{1}{N}\left(\sum_{k \in \mathcal{K}} e^{i \theta_{k}(n-m)}-\sum_{k \notin \mathcal{K}} e^{i \theta_{k}(n-m)}\right) .
\end{aligned}
$$

A few examples, that will be useful in the following, may help to understand the correspondence:

State 0. The vacuum $\left|\Psi^{(0)}\right\rangle=|0\rangle$, that corresponds to $\mathcal{K}^{(0)}=\emptyset$ and has associated a constant density $g^{(0)}(\theta)=-1$.

State 1. $\mathcal{K}^{(1)}=\{-N / 4+1, \ldots, N / 4-1, N / 4\}$ which corresponds to

$$
g^{(1)}(\theta)=\left\{\begin{aligned}
1 & \text { for } \theta \in(-\pi / 2, \pi / 2] \\
-1 & \text { for } \theta \notin(-\pi / 2, \pi / 2]
\end{aligned}\right.
$$




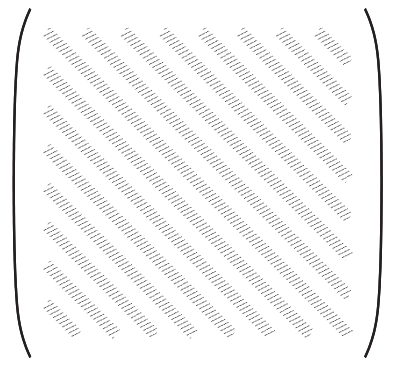

(a)

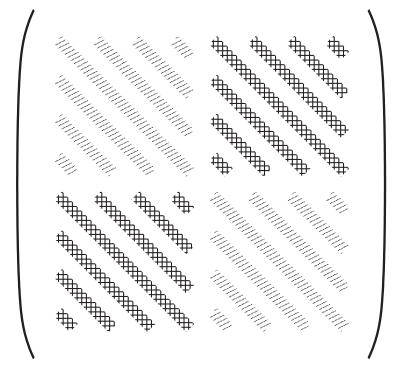

(b)
FIG. 2. By the diagonal bars in (a), we represent the defining property of a Toeplitz matrix: the entries along any subdiagonal parallel to the principal one are equal. Panel (b) represents a block matrix in which each block is Toeplitz but the full matrix is not.

State 2. $\mathcal{K}^{(2)}=\{-N / 2+2,-N / 2+4, \ldots, 0,2, \ldots, N / 2\}$, i.e., only even wave numbers are excited. The corresponding density is also constant, $g^{(2)}(\theta)=0$.

State 3. $\mathcal{K}^{(3)}=\{-N / 4+2,-N / 2+4, \ldots, 0,2, \ldots, N / 4\}$, i.e., even wave numbers between $-N / 4+2$ and $N / 4$ are excited. The corresponding density is

$$
g^{(3)}(\theta)=\left\{\begin{aligned}
0 & \text { for } \theta \in(-\pi / 2, \pi / 2], \\
-1 & \text { for } \theta \notin(-\pi / 2, \pi / 2] .
\end{aligned}\right.
$$

When subsystem $X$ is a single interval, i.e., it is composed of consecutive sites, we have an extra property that allows us to compute the asymptotic behavior of the Rényi entropy. In fact, in this case the matrix $V_{X}$ has all the entries of every subdiagonal parallel to the main one equal, as it is represented in Fig. 2(a). In other words, it is a diagonal-constant or Toeplitz matrix. Note that this property does not hold, in general, if there is some gap between two sites in $X$; Fig. 2(b) provides an example of this.

It turns out that there exist asymptotic formulas for computing the determinant of Toeplitz matrices and using them in (3) we can derive an expression for the entanglement entropy $[9,10]$. Applying the general results to our case of interest and taking a piecewise constant $g(\theta)$ with discontinuities at $\theta_{1}, \ldots, \theta_{R}$,

$$
g(\theta)=t_{r}, \quad \theta_{r-1}<\theta<\theta_{r},
$$

the entanglement entropy, when $X$ is an interval, reads

$$
S_{\alpha}(X)=A_{\alpha}|X|+B_{\alpha} \ln |X|+C_{\alpha}+\cdots,
$$

where the dots represent terms that vanish in the large $|X|$ limit. The coefficients depend only on $g(\theta)$, not on $X$, and their computation is described below.

In first place, the linear term is given by

$$
A_{\alpha}=\frac{1}{2 \pi} \int_{-\pi}^{\pi} f_{\alpha}(1, g(\theta)) d \theta .
$$

In order to compute $B_{\alpha}$ we introduce

$$
\omega_{r}(\lambda)=\frac{1}{2 \pi} \ln \left|\frac{\lambda-t_{r}}{\lambda-t_{r-1}}\right|,
$$

where the $t_{r}$ 's are taken from (6), and we define

$$
J_{\alpha}\left(r, r^{\prime}\right)=\frac{1}{2 \pi} \int_{t_{r-1}}^{t_{r}} \frac{d f_{\alpha}(1, \lambda)}{d \lambda} \omega_{r^{\prime}}(\lambda) d \lambda, \quad r, r^{\prime}=1, \ldots, R .
$$

Now

$$
B_{\alpha}=2 \sum_{r=1}^{R} J_{\alpha}(r, r),
$$

where the upper limit in the sum is the number of discontinuity points in $g(\theta)$.

The constant term requires one more definition

$$
I_{\alpha}(r)=\frac{1}{2 \pi i} \int_{t_{r-1}}^{t_{r}} \frac{d f_{\alpha}(1, \lambda)}{d \lambda} \ln \left[\frac{\Gamma\left(1 / 2-i \omega_{r}(\lambda)\right)}{\Gamma\left(1 / 2+i \omega_{r}(\lambda)\right)}\right] d \lambda,
$$

where $\Gamma$ stands for the $\Gamma$ function. From it we can write

$$
C_{\alpha}=\sum_{r=1}^{R} I_{\alpha}(r)-\sum_{1 \leqslant r \neq r^{\prime} \leqslant R} \ln \left[2-2 \cos \left(\theta_{r}-\theta_{r^{\prime}}\right)\right] J_{\alpha}\left(r, r^{\prime}\right) .
$$

The previous coefficients have been derived in [9] using the Fisher-Hartwig conjecture for Toeplitz determinants [27], probed in our case by Basor [28,29]. In [9] we also perform the numeric computation of the entropy and compare it with the asymptotic results to show the perfect agreement between both calculations.

The results for the states defined before are the following:

State 0 . In this case $g^{(0)}(\theta)=-1$ and as we have $f_{\alpha}(1,-1)=0$ the linear coefficient vanishes. On the other hand, $g^{(0)}$ has no discontinuities and therefore $B_{\alpha}^{(0)}$ and $C_{\alpha}^{(0)}$ vanish and the entanglement entropy $S_{\alpha}^{(0)}=0$, which can be obtained directly by noticing that $\left|\Psi^{(0)}\right\rangle$ is separable.

State 1. As it is well known [10] this state can be interpreted as the ground state of a one-dimensional, local, critical theory. Therefore, the results from conformal field theory apply and we should have [16]

$$
S_{\alpha}^{\text {crit }}(X)=\frac{c}{6} \frac{\alpha+1}{\alpha} \ln |X|+C_{\alpha}+\cdots,
$$

where $c$ is the central charge of the underlying conformal field theory and $C_{\alpha}$ is a constant that depends on the details of the theory (nonuniversal). On the other hand, if we apply our general result and due to the fact that $f_{\alpha}(1, \pm 1)=0$ we have $A_{\alpha}^{(1)}=0$. Also the logarithmic coefficient can be computed analytically to give $B_{\alpha}^{(1)}=(\alpha+1) /(6 \alpha)$, while the constant term does not have a simple expression for general $\alpha$. Putting it all together we have

$$
S_{\alpha}^{(1)}(X)=\frac{\alpha+1}{6 \alpha} \ln |X|+C_{\alpha}^{(1)}+\cdots .
$$

That agrees with (12) for a central charge $c=1$.

State 2. If we write $\left|\Psi^{(2)}\right\rangle$ in the basis of positions we have

$$
\left|\Psi^{(2)}\right\rangle=\prod_{n=1}^{N / 2} \frac{1}{\sqrt{2}}\left(a_{n}^{\dagger}+a_{n+N / 2}^{\dagger}\right)|0\rangle
$$

Therefore it is easy to compute, exactly, the entanglement entropy for any subsystem. In particular if $X$ is an interval 
of size smaller than $N / 2$ the reduced density matrix is proportional to the identity

$$
\rho_{X}=2^{-|X|} I
$$

and therefore

$$
S_{\alpha}^{(2)}(X)=|X| \ln 2,
$$

which is independent of $\alpha$ and is the largest possible entropy for a mixed state in a Hilbert space of dimension $2^{|X|}$. If we derive the coefficients of the expansion according to (8), (9), and (11) we get $A_{\alpha}^{(2)}=f_{\alpha}(1,0)=\ln 2$ and, as we do not have any discontinuity, $B_{\alpha}^{(2)}=C_{\alpha}^{(2)}=0$. Finally, the expansion leads to (13) that is exact in this case.

State 3. The entanglement entropy for this state combines the features of the two previous ones: it has a nonzero linear coefficient as $g(\theta)$ is different from \pm 1 in some interval and it has discontinuities which give rise to the logarithmic and constant coefficients. The linear term is easily computed to give $A_{\alpha}^{(2)}=\ln 2 / 2$ and for integer $\alpha \geqslant 2$ we have [9],

$$
B_{\alpha}^{(2)}=\frac{\alpha+1}{24 \alpha}-\frac{1}{2 \pi^{2}(\alpha-1)} \sum_{l=1}^{\alpha}\left(\ln \sin \frac{(2 l-1) \pi}{2 \alpha}\right)^{2},
$$

while for $\alpha=1$

$$
B_{1}=\frac{1}{8}-\frac{1}{2}\left(\frac{\ln 2}{\pi}\right)^{2}
$$

In this paper we want to go one step further and discuss the case when $X$ is made out of several intervals. Our goal is to derive asymptotic formulas for the entanglement entropy similar to (7). In this case, however, we cannot use the Fisher-Hartwig formula and we will approach the problem by using the results from conformal field theory and performing numeric computations. Later on we will be able to derive a generalization of Fisher-Hartwig conjecture that covers the case of several intervals.

\section{TWO DISJOINT INTERVALS}

As it was mentioned before, if the set $X$ is composed of noncontiguous sites, for instance the union of two separate intervals $X=X_{1} \cup X_{2}$ with $X_{1}$ and $X_{2}$ made out of contiguous sites, the matrix $V_{X}$ is not of the Toeplitz type any more. This is shown pictorially in Fig. 2(b), where we represent the fact that while all four submatrices are Toeplitz, the full matrix is not: except for the main diagonal all the others have two kinds of entries.

In this situation we cannot apply the Fisher-Hartwig conjecture and we should try to get insights on the behavior of the entanglement entropy from a different source.

If the state we consider corresponds to the ground state of a critical, local, one-dimensional system (like it happens for the state 1) we can use the conformal invariance of the theory, that follows from the absence of a fundamental length (zero mass gap). This powerful symmetry determines to some extent the behavior of the entanglement entropy.
In this case [23] if we take $X=\left[u_{1}, v_{1}\right] \cup\left[u_{2}, v_{2}\right]$ with $u_{1}<v_{1}<u_{2}<v_{2}$, global conformal invariance leads to

$$
\begin{aligned}
\operatorname{Tr} \rho_{X}^{\alpha} \sim & K_{\alpha}\left(\frac{\left(v_{1}-u_{1}\right)\left(v_{2}-u_{2}\right)\left(v_{2}-u_{1}\right)\left(u_{2}-v_{1}\right)}{\left(u_{2}-u_{1}\right)\left(v_{2}-v_{1}\right)}\right)^{4 \alpha \Delta_{\alpha}} \\
& \times \mathcal{G}(y),
\end{aligned}
$$

where

$$
y=\frac{\left(u_{2}-v_{1}\right)\left(v_{2}-u_{1}\right)}{\left(u_{2}-u_{1}\right)\left(v_{2}-v_{1}\right)}
$$

is the cross ratio $\left(u_{1}, v_{1} ; u_{2}, v_{2}\right)$ which is invariant under the linear fractional transformations $z \mapsto(a z-b) /(d-c z), \mathcal{G}$ is a nonuniversal function that depends on the details of the theory (see [23]),

$$
\Delta_{\alpha}=\frac{c}{24}\left(1-\frac{1}{\alpha^{2}}\right)
$$

is the conformal dimension of the insertions, and, finally, $K_{\alpha}$ is a constant that will be fixed below.

In [26] it is shown that for the ground state of critical free fermions (as is our case) $\mathcal{G}=1$. Then if we compute the Rényi entropy we obtain

$$
\begin{aligned}
S_{\alpha}(X)= & \frac{c}{6} \frac{\alpha+1}{\alpha} \ln \frac{\left(v_{1}-u_{1}\right)\left(v_{2}-u_{2}\right)\left(v_{2}-u_{1}\right)\left(u_{2}-v_{1}\right)}{\left(u_{2}-u_{1}\right)\left(v_{2}-v_{1}\right)} \\
& +\frac{1}{1-\alpha} \ln K_{\alpha}+\cdots .
\end{aligned}
$$

In order to determine the constant on the right we can take the limit of large separation between $X_{1}$ and $X_{2}$ in which case the entropy should go to the sum of the entropy of every interval. Therefore the constant on the right of (17) should be twice the constant for a single interval $C_{\alpha}$ that we determined in the previous section.

In summary, for a critical, local theory for which $g(\theta)=$ \pm 1 , like the state 1 , we should have

$$
\begin{aligned}
S_{\alpha}^{(1)}(X)= & B_{\alpha}^{(1)} \ln \frac{\left(v_{1}-u_{1}\right)\left(v_{2}-u_{2}\right)\left(v_{2}-u_{1}\right)\left(u_{2}-v_{1}\right)}{\left(u_{2}-u_{1}\right)\left(v_{2}-v_{1}\right)} \\
& +2 C_{\alpha}^{(1)}+\cdots,
\end{aligned}
$$

where the constants are those determined in the previous section for a single interval. We have checked the expression against numerical results and it completely agrees.

Another insight on the problem can be gained by considering the state 2 . In this case, we can compute the entropy exactly and, provided $\left|v_{2}-u_{1}\right|<N / 2$ we have that the reduced density matrix is again proportional to the identity and

$$
S_{\alpha}^{(2)}(X)=|X| \ln 2
$$

where, in this case, $|X|=\left|v_{1}-u_{1}\right|+\left|v_{2}-u_{2}\right|$. It should be noticed that the coefficient that multiplies the size of the subsystem coincides with that for a single interval.

If we put together the two previous results we conjecture the following expression for the Rényi entanglement entropy of two disjoint intervals for a general state:

$$
\begin{aligned}
S_{\alpha}(X)= & A_{\alpha}|X| \\
& +B_{\alpha} \ln \frac{\left(v_{1}-u_{1}\right)\left(v_{2}-u_{2}\right)\left(v_{2}-u_{1}\right)\left(u_{2}-v_{1}\right)}{\left(u_{2}-u_{1}\right)\left(v_{2}-v_{1}\right)} \\
& +2 C_{\alpha}+\cdots,
\end{aligned}
$$



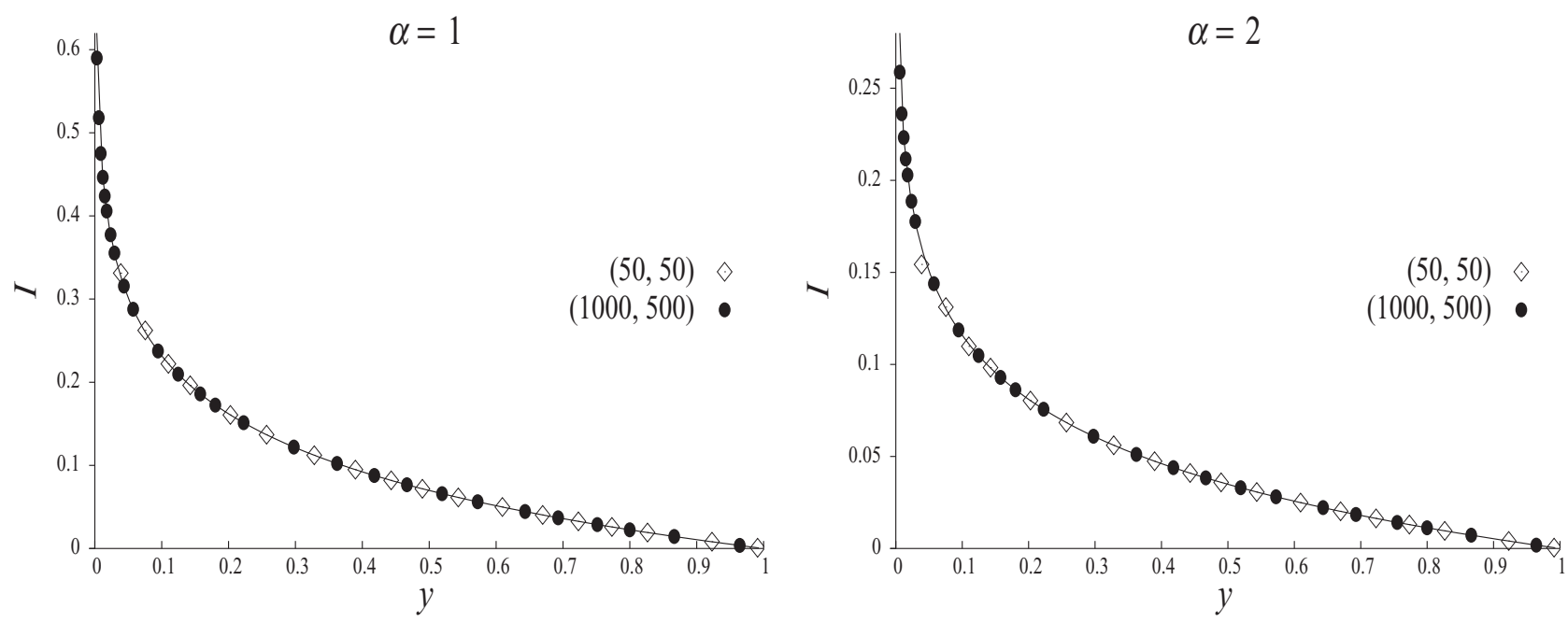

FIG. 3. Two blocks mutual information when $\alpha=1$ (left panel) and $\alpha=2$ (right panel) as a function of $y$ for state 3 . With $\diamond$ we represent the numerical value for two blocks made out of 50 sites each, varying their separation from 1 up to 500 sites. The $\bullet$ corresponds to two blocks of lengths 1000 and 500 sites, separating each other between 1 and 1000 sites. The continuous line depicts the function (20) with the $B_{\alpha}$ evaluated from the Fisher-Hartwig conjecture.

where the coefficients $A_{\alpha}, B_{\alpha}, C_{\alpha}$ are those determined in (8), (9), and (11) for a single interval. The expression (19) should be valid in the thermodynamic limit, while the dots stand for contributions that vanish when $\left|v_{i}-u_{j}\right| \rightarrow \infty$ for $i, j=1,2$.

In the next section we will investigate numerically the validity of our conjecture.

\section{NUMERICAL RESULTS}

In order to check the accuracy of the previous expression it will be useful to introduce the so-called mutual information $I_{\alpha}\left(X_{1}, X_{2}\right)$ defined by

$$
I_{\alpha}\left(X_{1}, X_{2}\right)=S_{\alpha}\left(X_{1}\right)+S_{\alpha}\left(X_{2}\right)-S_{\alpha}(X) .
$$

From (19) we obtain the simple expression

$$
I_{\alpha}\left(X_{1}, X_{2}\right)=-B_{\alpha} \ln y
$$

where $y$ is the cross ratio in Eq. (16). The logarithmic coefficient $B_{\alpha}$ is obtained from the Fisher-Hartwig conjecture for the expansion of the entropy for a single interval, as it is discussed in Sec. II.

We perform numerical calculations for state 3 choosing an infinite chain, $N \rightarrow \infty$, when $\alpha=1$ (von Neumann entropy) and $\alpha=2$. Since the Wick decomposition is satisfied, they can be carried out diagonalizing the correlation matrix $V_{X}(4)$ for this configuration and then applying the first equality of (3). As we have discussed before, this implies an impressive simplification $\left(V_{X}\right.$ has dimension $|X|$ while $\left.\rho_{X}, 2^{|X|}\right)$ which we need to explore the asymptotic behavior of the mutual information. In fact, in the following numerical computation we have covered values of $|X|$ from 100 to 5500 . Notice that these values would be absolutely out of reach in a direct computation using $\rho_{X}$, with dimension $2^{5500}$.

For the diagonalization, we have employed the corresponding routine for real symmetric matrices included in the GNU Scientific Library [30] for $\mathrm{C}$, which works in double precision.
Dots in Fig. 3 represent the numerical results for two different sizes of the blocks. The continuous line stands for the analytic candidate (20). Remember for state $3 B_{\alpha}$ is given by the expression (14) for an integer $\alpha \geqslant 2$. For $\alpha=2$ it leads,

$$
B_{2}^{(3)}=\frac{1}{16}-\frac{1}{4}\left(\frac{\ln 2}{\pi}\right)^{2}=0.050330 \ldots
$$

Notice that for this configuration, we have

$$
B_{1}^{(3)}=2 B_{2}^{(3)}=0.100660 \ldots
$$

There is an excellent agreement between the numerical results and the analytical expression (20) we have proposed. From the plots it is also apparent that the mutual information only depends on the cross ratio of the involved distances. We have also performed the computations with other block lengths and different states, finding the same accordance with the theoretical prediction.

\section{SEVERAL INTERVALS AND A GENERALIZATION OF THE FISHER-HARTWIG CONJECTURE}

The results obtained in the previous section for two intervals can be immediately generalized to the case of $p$ disjoint intervals. Namely, consider

$$
X=\bigcup_{i=1}^{p}\left[u_{i}, v_{i}\right], \quad u_{i}<v_{i}<u_{i+1}
$$

then, keeping in mind the results of conformal field theory and (19), it is natural to write

$$
\begin{aligned}
S_{\alpha}(X)= & A_{\alpha} \sum_{i=1}^{p}\left(v_{i}-u_{i}\right)+B_{\alpha} \ln \frac{\prod_{i, j=1}^{p}\left|u_{i}-v_{j}\right|}{\prod_{j>i}\left(u_{j}-u_{i}\right)\left(v_{j}-v_{i}\right)} \\
& +p C_{\alpha},
\end{aligned}
$$


where the last two terms are taken directly from the conformal field expressions and the first one reflects the extensivity of the linear term. Like before, this expression should be valid in the thermodynamic limit, and the dots represent terms that vanish when $\left|u_{i}-v_{j}\right| \rightarrow \infty, i, j=1, \ldots, p$.

In order to check (21) it will be useful to introduce the analog of the mutual information for $p$ intervals, given by

$$
\begin{aligned}
I_{\alpha}\left(\left[u_{1}, v_{1}\right], \ldots,\left[u_{p}, v_{p}\right]\right)= & \sum_{i=1}^{p} S_{\alpha}\left(\left[u_{i}, v_{i}\right]\right) \\
& -S_{\alpha}\left(\bigcup_{1=1}^{p}\left[u_{i}, v_{i}\right]\right) .
\end{aligned}
$$

Note that this is different from the tripartite mutual information of [31]. Actually according to our results the latter vanishes in the asymptotic limit.

If (21) is correct we should have

$$
I_{\alpha}\left(\left[u_{1}, v_{1}\right], \ldots,\left[u_{p}, v_{p}\right]\right)=-B_{\alpha} \ln \prod_{i<j} y_{i j},
$$

where

$$
y_{i j}=\frac{\left(u_{j}-v_{i}\right)\left(v_{j}-u_{i}\right)}{\left(u_{j}-u_{i}\right)\left(v_{j}-v_{i}\right)}
$$

is the cross ratio of $\left(u_{i}, v_{i} ; u_{j}, v_{j}\right)$.

In order to verify (23) we have computed (22) numerically for three and four intervals for state 3 with different sizes and distances for the intervals. The results are shown in Fig. 4 together with the conjectured behavior stated in (23). The agreement is extraordinary.

The expression for the entropy in Eq. (21) can also be written as a combination of that for single intervals,

$$
\begin{aligned}
S_{\alpha}(X)= & \sum_{i \geqslant j} S_{\alpha}\left(\left[u_{j}, v_{i}\right]\right)+\sum_{i<j}\left(S_{\alpha}\left(\left[v_{i}, u_{j}\right]\right)-S_{\alpha}\left(\left[v_{i}, v_{j}\right]\right)\right. \\
& \left.-S_{\alpha}\left(\left[u_{i}, u_{j}\right]\right)\right) .
\end{aligned}
$$

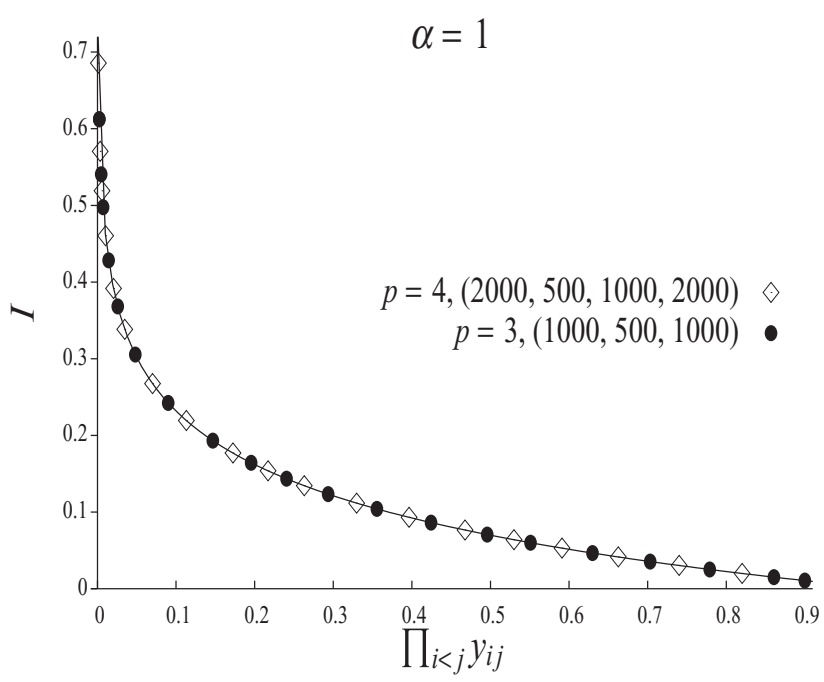

It is not difficult to show that combining the expression above and (7) we derive Eq. (21). However (24) has the virtue of showing more clearly the possible origin of our result for several intervals, as we will see below. An expression similar to (24) for the ground state of local critical theories has been derived in [32] applying the holographic principle. See also Ref. [31].

In the computation of the entropy for a single interval a key step was to use the asymptotic expansion of the Toeplitz determinant which appears in the integrand of (3) and is well known in the literature. However, as it was noticed before, for more than one interval the correlation matrix is not Toeplitz any more but it is a principal submatrix of a Toeplitz matrix, as it is depicted in Fig. 2(b).

On the other hand, as is made explicit in (3), $S_{\alpha}(X)$ depends linearly on the logarithm of the determinant of this submatrix. Therefore, the relation (24) can be derived from an analogous property for the determinants of principal submatrices of a Toeplitz matrix.

In order to formulate the conjecture, consider a general Toeplitz matrix $T$ with piecewise smooth symbol $g(\theta)$ and for any set of indices $X$ define $D(X)=\operatorname{det}\left(T_{n m}\right), n, m \in X$. Then, the property for the determinant of the principal submatrix that we hypothesize can be stated as follows.

\section{Conjecture.}

$$
D\left(\bigcup_{i=1}^{p}\left[u_{i}, v_{i}\right]\right) \simeq \prod_{i} D\left(\left[u_{i}, v_{i}\right]\right) \prod_{i<j} \frac{D\left(\left[u_{i}, v_{j}\right]\right) D\left(\left[v_{i}, u_{j}\right]\right)}{D\left(\left[u_{i}, u_{j}\right]\right) D\left(\left[v_{i}, v_{j}\right]\right)}
$$

where $\simeq$ stands for the equality of the asymptotic behavior when $\left|v_{i}-u_{j}\right| \rightarrow \infty$, for $i, j=1, \ldots, p$. Notice that all determinants on the right-hand side are of the Toeplitz type and therefore, using the Fisher-Hartwig conjecture, (25) allows the computation of the scaling of general principal submatrices of

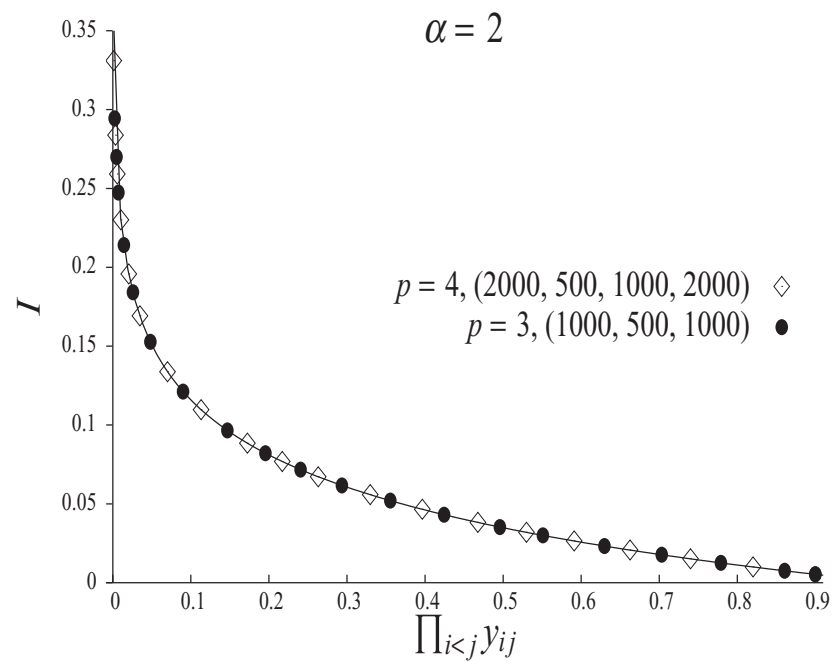

FIG. 4. Mutual information, for state 3, of three $(\bullet)$ and four $(\diamond)$ blocks as a function of the product of the possible cross ratios $y_{i j}$. For $p=3$, we have chosen two intervals of 1000 sites and one of 500 sites which is separated from one of the former by 1500 sites, while the remaining distance is modified from 1 up to 99000 sites. For $p=4$, we take intervals of lengths 2000, 500, 1000, 2000. The distance between the first couple is 1500 sites, the break between the smallest blocks is also fixed, 5000 sites, whereas the other one is increased between 1 and 99000 sites. 


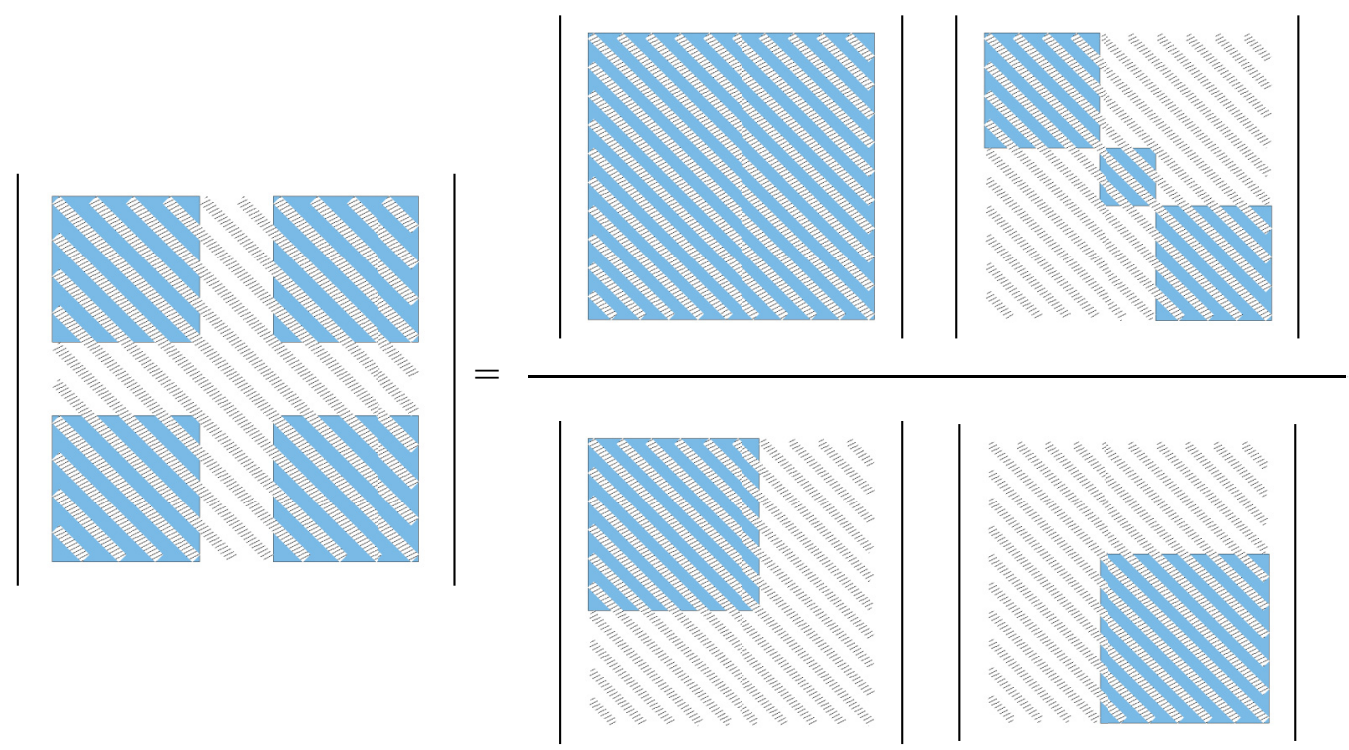

FIG. 5. (Color online) Graphical representation of conjecture (25) for $p=2$. In the left-hand side we represent the determinant of the shadowed submatrix that, in general, is not Toeplitz. In the right-hand side, however, the determinants of the shadowed submatrices are of the Toeplitz type (or product of these).

a Toeplitz matrix. Formula (25) is depicted graphically, for $p=2$, in Fig. 5 .

Of course, the previous conjecture has been checked indirectly when we have computed the entropy of several intervals, but we think it is worth studying it for a more general, piecewise smooth symbol. We choose the following:

$$
g(\theta)= \begin{cases}\frac{1}{4}(3+\sin \theta), & \theta \in(-\pi, 0], \\ \frac{1}{4}(3+\cos \theta), & \theta \in(0, \pi],\end{cases}
$$

from which it is easy to compute the corresponding matrix:

$$
\begin{aligned}
T_{n m} & =\frac{1}{2 \pi} \int_{-\pi}^{\pi} g(\theta) e^{i(n-m) \theta} d \theta \\
& = \begin{cases}0, & \text { for } n-m \text { odd }, \\
\frac{1}{4 \pi} \frac{(n-m) i+1}{(n-m)^{2}-1}+\frac{3}{4} \delta_{n m}, & \text { for } n-m \text { even. }\end{cases}
\end{aligned}
$$

We check (25) in this particular case for $p=2$, studying the analog for determinants of mutual information,

$$
\begin{aligned}
I_{D}\left(\left[u_{1}, v_{1}\right] \cup\left[u_{2}, v_{2}\right]\right)= & \ln D\left(\left[u_{1}, v_{1}\right]\right)+\ln D\left(\left[u_{2}, v_{2}\right]\right) \\
& -\ln \left(D\left[u_{1}, v_{1}\right] \cup\left[u_{2}, v_{2}\right]\right) .
\end{aligned}
$$

Then, applying the Fisher-Hartwig conjecture for the Toeplitz submatrices which appear in (25), we should have

$$
I_{D}\left(\left[u_{1}, v_{1}\right] \cup\left[u_{2}, v_{2}\right]\right)=-B_{D} \ln y,
$$

where the coefficient $B_{D}$ can be obtained analytically from the general expression (9). For this particular case it is

$$
\begin{aligned}
B_{D}= & \frac{1}{2 \pi^{2}}\left[\int_{3 / 4}^{1} \frac{1}{\lambda} \ln \left|\frac{\lambda-1}{\lambda-3 / 4}\right| d \lambda\right. \\
& \left.+\int_{1 / 2}^{3 / 4} \frac{1}{\lambda} \ln \left|\frac{\lambda-3 / 4}{\lambda-1 / 2}\right| d \lambda\right]=0.0062607 \ldots
\end{aligned}
$$

In Fig. 6, we represent by dots the numerical value of $I_{D}$ while the solid line represents the logarithmic dependence (27) predicted by our conjecture, with the coefficient computed above.

The agreement between our conjecture and the numerical results is certainly remarkable. Due to the asymptotic nature of our formulas, the accordance with the numerical result should be poorer when the separation between the intervals is only of a few sites. This is especially striking when we study the determinant of two small subsets as we can see in Fig. 6.

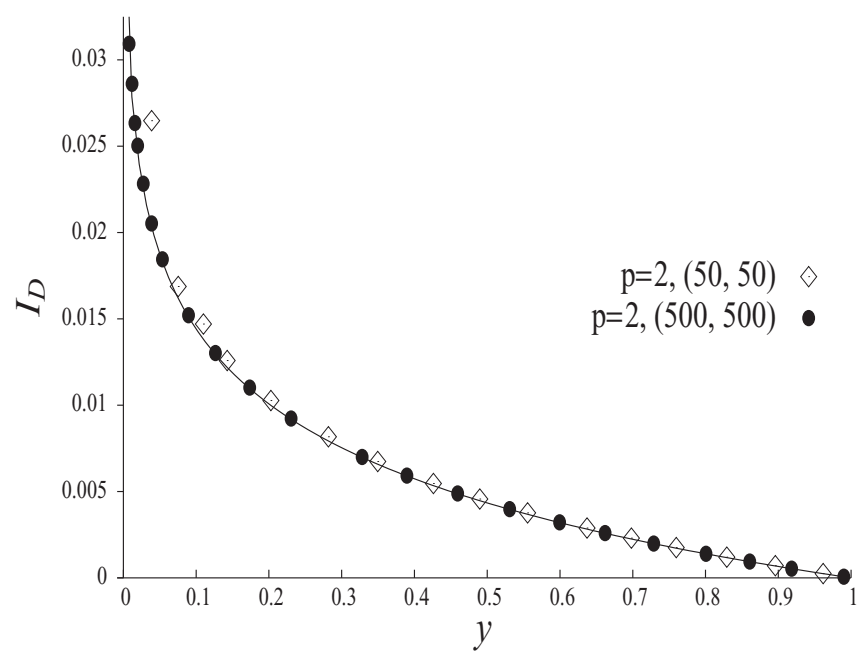

FIG. 6. Analog of mutual information for determinants, (26), against the cross ratio of $\left(u_{1}, v_{1} ; u_{2}, v_{2}\right)$. The $\diamond$ represents the numerical results for two subsets of size $\left|u_{1}-v_{1}\right|=\left|u_{2}-v_{2}\right|=50$ while the gap between them, $\left|v_{1}-u_{2}\right|$, varies between 1 and 200. The $\bullet$ corresponds to two subsets of length 500 separated by a distance between 1 and 4500 . The continuous line is the conjectured analytical expression of (27). 


\section{CONCLUSIONS}

In this paper we have studied the entanglement between two subsystems made out of several disjoint intervals for the eigenstates of a unidimensional fermionic chain described by a free, translational invariant Hamiltonian.

Similarly to the case of a single interval, since these states satisfy the Wick decomposition, we can compute the Rényi entanglement entropy employing the two-point correlation matrices restricted to one of the subsystems. This reduces the complexity which, in principle, grows exponentially with the size of our subsystem. On the other hand, for two or more disjoint blocks, these correlations are no more Toeplitz matrices (in fact, they are block matrices where each block is Toeplitz). Hence, the Fisher-Hartwig expansion does not hold.

Therefore, we have been forced to resort to a different strategy in order to gain understanding on the behavior of the entropy for several intervals. One of the sources for our intuition comes from conformal field theory, that can be applied to the ground state of local, gapless theories. The other source is the opposite: nonlocal theories (ladders) with a mass gap. In both cases there is an alternative way of computing the entropy: using conformal invariance in the first instance and by direct computation in the second case.

Extending these partial results, we propose a general asymptotic expansion for an arbitrary translational invariant state. We have checked that it perfectly matches with the numerical value for different states and several block numbers and sizes.

Finally, from this result we can conjecture the solution for a more general problem: the asymptotic behavior of the determinant of general principal submatrices of a Toeplitz matrix. Our result relates the determinant of our submatrix to the product of several others of the Toeplitz type, which, combined with the Fisher-Hartwig theorem, provides an asymptotic scaling for this kind of determinants. We have numerically verified our conjecture for a particular Toeplitz matrix with a piecewise smooth symbol.

One of the motivations for working with a chain of spinless fermions is its relation with spin chains. In the general case, however, the resulting Hamiltonian for the fermionic chain, although it is still quadratic, does not preserve the total fermionic number. Then, the two-point function involves more coefficients and the resulting matrix is of the, so-called, block Toeplitz type. It would be nice to extend our results for general stationary states to these systems.

\section{ACKNOWLEDGMENTS}

Research was partially supported by Grants No. 2012E24/2, DGIID-DGA and No. FPA2012-35453, MINECO (Spain). F.A. is supported by FPI Grant No. C070/2014, DGIID-DGA.
[1] E. Schrödinger, Proc. Cambridge Philos. Soc. 31, 555 (1935); 32, 446 (1935).

[2] P. Calabrese, J. Cardy, and B. Doyon, J. Phys. A: Math. Theor. 42, 500301 (2009).

[3] J. I. Latorre and A. Riera, J. Phys. A: Math. Theor. 42, 504002 (2009).

[4] P. Calabrese and J. Cardy, J. Phys. A: Math. Theor. 42, 504005 (2009).

[5] H. Casini and M. Huerta, J. Phys. A: Math. Theor. 42, 504007 (2009).

[6] S. N. Solodukhin, Living Rev. Relativity 14, 8 (2011).

[7] T. Nishioka, S. Ryu, and T. Takayanagi, J. Phys. A: Math. Theor. 42, 504008 (2009).

[8] M. B. Plenio and S. Virmani, Quantum Inf. Comput. 7, 1 (2007).

[9] F. Ares, J. G. Esteve, F. Falceto, and E. Sánchez-Burillo, J. Phys. A: Math Theor. 47, 245301 (2014).

[10] B. Q. Jin and V. E. Korepin, J. Stat. Phys. 116, 79 (2004).

[11] Z. Kádar and Z. Zimborás, Phys. Rev. A 82, 032334 (2010).

[12] V. Eisler and Z. Zimborás, Phys. Rev. A 89, 032321 (2014).

[13] J. P. Keating and F. Mezzadri, Phys. Rev. Lett. 94, 050501 (2005).

[14] V. Alba, M. Fagotti, and P. Calabrese, J. Stat. Mech. (2009) P10020.

[15] C. Holzhey, F. Larsen, and F. Wilczek, Nucl. Phys. B 424, 443 (1994).

[16] P. Calabrese and J. Cardy, J. Stat. Mech. (2004) P06002.
[17] G. Vidal, J. I. Latorre, E. Rico, and A. Kitaev, Phys. Rev. Lett. 90, 227902 (2003).

[18] F. C. Alcaraz and M. S. Sarandy, Phys. Rev. A 78, 032319 (2008).

[19] F. C. Alcaraz, M. I. Berganza, and G. Sierra, Phys. Rev. Lett. 106, 201601 (2011); M. I. Berganza, F. C. Alcaraz, and G. Sierra, J. Stat. Mech. (2012) P01016.

[20] I. Peschel, J. Phys. A: Math. Gen. 36, L205 (2003).

[21] M. Caraglio and F. Gliozzi, J. High Energy Phys. 11 (2008) 076.

[22] S. Furukawa, V. Pasquier, and J. Shiraishi, Phys. Rev. Lett. 102, 170602 (2009).

[23] P. Calabrese, J. Cardy, and E. Tonni, J. Stat. Mech. (2009) P11001.

[24] P. Facchi, G. Florio, C. Invernazzi, and S. Pascazio, Phys. Rev. A 78, 052302 (2008).

[25] M. Fagotti, Europhys. Lett. 97, 17007 (2012).

[26] H. Casini and M. Huerta, Class. Quantum Grav. 26, 185005 (2009).

[27] M. E. Fisher and R. E. Hartwig, Adv. Chem. Phys. 15, 333 (1968).

[28] E. L. Basor, Indiana Math. J. 28, 975 (1979).

[29] E. L. Basor and K. E. Morrison, Linear Algebra Appl. 202, 129 (1994).

[30] M. Galassi et al., GNU Scientific Library Reference Manual, 3rd ed., http://www.gnu.org/software/gsl/

[31] H. Casini and M. Huerta, J. High Energy Phys. 03 (2009) 048.

[32] V. Hubeny and M. Rangamani, J. High Energy Phys. 03 (2008) 006. 